\title{
The application of Artificial Intelligence to Ophthalmology: A bibliometric study (2000-2021)
}

\author{
Han Wang ${ }^{1,2,3}$, Xiaoshu Zhou ${ }^{2}$, Wencai Du ${ }^{4}$, and Lina Huang ${ }^{5 *}$ \\ ${ }^{1}$ Institute of Data Science, City University of Macau, Macau 999078, \\ D20092100037@cityu.mo. \\ ${ }^{2}$ School of Computer, Beijing Institute of Technology Zhuhai, China 519000, \\ 20251@bitzh.edu.cn, 402659826@qq.com. \\ ${ }^{3}$ Zhuhai Institute of Advanced Technology Chinese Academy of Sciences, China \\ 519000, wanghan@ziat.ac.cn. \\ ${ }^{4}$ University of Saint Joseph, Macau 999078; Geogedu@usj.edu.mo. \\ 5Jinan University Affiliated Shenzhen Aier Eye Hospital, China 518000; lina_h@126.com. \\ Corresponding author: \\ Lina Huang ${ }^{5}$ \\ Email address: lina_h@126.com.
}

\begin{abstract}
Background. Artificial Intelligence (Al) is an advanced technology for the latest 20 years. Machine learning (ML) and deep learning (DL) are the major innovations for Al, which has been applied for multiple fields. Ophthalmology has become to be one of the most significant disciplines for human healthcare.

Methodology. This study utilizes methods of text mining and bibliometric analysis to explore applications of Al to ophthalmology. 179 related articles from Web of Science (WOS) and 96 papers from China National Knowledge Infrastructure (CNKI) are explored during the period of 2000 to 2021. A descriptive analysis of major trends, journal releasing, topic mapping and quotation relationships is implemented in this paper. Leading authors, journals, institutions, nations and references in the related research are identified.

Results. Findings show that the application of Al technologies in ophthalmologic diagnosis with optical coherence tomography (OCT) fundus images is the core topic for this area's studies, especially for diabetic retinopathy (DR), aged macular degeneration (AMD) and glaucoma. It is also be predicted as the core direction over the recent years. Besides, The USA, England and China is the most competitive countries in this scientific filed. Journals of Ophthalmology, Investigative Ophthalmology and Visual Science, Eye, Acta Ophthalmologica and Scientific Reports are the top five journal related to the research area. There is a significant difference between WOS and CNKI databases pertaining to the application of Artificial Intelligence $(\mathrm{Al})$ to ophthalmology, especially for the historic development, topic mapping and discipline category. Finally, the potential academic value of interdisciplinary subject of "Al in Ophthalmology" and tradition Chinese medicine (TRM) is discussed. Limitations and suggestions for the future research is indicated at the end of this paper.

Keywords.Artificial Intelligence, Ophthalmology, Bibliometric Study, CiteSpace, Machine Learning, Deep Learning.
\end{abstract}

\section{INTRODUCTION}

The eye disease is regarded as one of the most significant healthcare problems, such as diabetic retinopathy (DR), age-related macular degeneration (AMD), glaucoma, retinal vein occlusion (RVO), retinal artery occlusion (RAO), retinal detachment (RD), epimacular membrane (EM), fundus tumors (FT), papilledema and diabetic macular edema (DME) (Yang et al. (2021); Shao et al. (2021); Cen et al. (2021)). Fundus diseases are the major cause for vision loss. For instance, DR is estimated as the most cause for blindness of adults (Dai et al. (2021)). The shortage of ophthalmologists and diagnose services are the central issue for lesion detection (Cen et al. (2021)). Thus, a computer-aided detection solution plays a vital role for 
ophthalmic diagnosis.

The enhancement of Artificial Intelligence (AI) presents a valuable potential for human healthcare in resent years, which exhibits a vital advantage in ophthalmology at the clinical level (Scheetz et al. (2021)). Optical coherence tomography (OCT) technology is regarded as a breakthrough for choroidal fundus image acquirement technology, which is a foundation of AI diagnose based on fundus images (Marta et al. (2021)). Machine learning (ML) and deep learning (DL) algorithms are wildly developed and applied for fundus diseases detection (Ran et al. (2021)). Studies related to AI-based applications in ophthalmology are constantly emerging and developing in the latest 20 years.

Scientometrics is a research method based on field-oriented scientific literatures. This method has been considered as one of the most significant approaches for literature analyzing in multiple areas (Sooryamoorthy (2020)). CiteSpace is one of the most popular tools for the bibliometric study. It (Wu et al. (2020)) is functioned by analyzing and measuring the topic, author, institution, journal, develop trend, etc. A systematic literature analysis contributes a reference for related scholars to understand specific researches in the perspectives of the present state and the future direction.

This article delivered a bibliometric study on applications of AI technologies to ophthalmology, where ML and DL algorithms are included. The structure of this paper is concluded as the following. The second section introduces the method of this study, where data sources and analysis methods are involved. Results and the discussion are delivered in the third and fourth section respectively. The conclusion is presented in the final section.

\section{METHODOLOGY}

\section{Data sources}

Scientific articles in English are obtained from Web of Science (WOS). Chinese papaers are collected from China National Knowledge Infrastructure (CNKI). Search string is defined as: TS= ("AI" OR "artificial intelligen*” OR "deep learning" OR "machine learning") AND TS=(“Ophthalmolog*"). Time span is set as 1st January 2000 to 10th October 2021.

\section{Analysis methods}

TXT results from WOS and CNKI are input into the software of CiteSpace. The version of this software is 5.7.R3,64 bit for the Window operation system. Analysis of authors, institution, countries, keywords, categories and journals are conducted on the collected data. A process of visualization and comparable analysis is implemented on the results.

\section{RESULTS}

With 179 articles from WOS and 96 papers from CNKI, the development trend of the application of AI in ophthalmology research are explored from 2000 to 2021. Authors, institutions, journals, countries and hot-spots are descriptively analyzed in this section.

\section{Analysis of the trends in the literature}

The Fig. 1 shows the developing history on "AI applied to Ophthalmology" from 2000 to 2020. Numbers of publications grew significantly in the period of 2014 to 2020 for WOS, and 2017 to 2020 for CNKI. From 2014 to 2020, 144 articles are recorded in WOS database, which is approximately 4 times more than the period of 2000 to 2014. It presents a similar increase trend for CNKI. In 2017, 9 papers were published in CNKI, it is increased to 72 publications in 2020, which is 8 times of 2017. The amount is predicted to be continuously increasing in the recent years.

\section{Analysis of the countries, authors, institutions, journals and cited references}

A country distribution figure (Fig. 2) is generated based on WOS database in CiteSpace. 19 notes and 25 links (Fig. 2.) are given in this map, which represents that 52 countries and 25 collaboration are involved. Top 14 countries are listed in Tab 1 based on the amount of the publications, where USA (centrality $=0.63$, count=38), England (centrality $=0.28$, count $=14$ ) and China (centrality $=0.24$, count $=11$ ) are regarded as the top 3 countries based on centrality and co-occurrence. Fig. 2. indicates that the strongest cooperation is between Germany and Netherlands. 
60 History Trend of WOS and CNKI

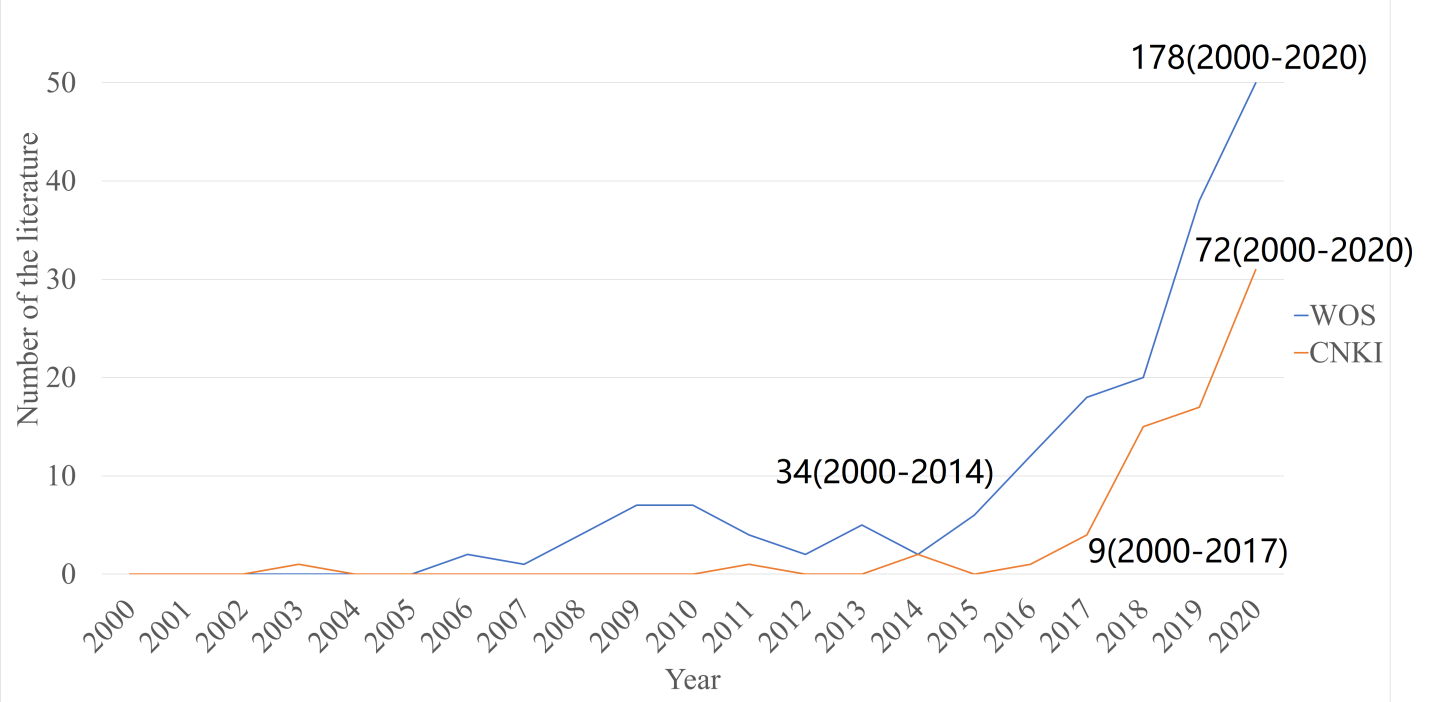

Figure 1. History trend analysis of WOS and CNKI.

The social networks of authors from WOS (a) and CNKI(b) display in Fig. 3.21 nodes and 19 links for WOS, and 239 nodes and 613 for CNKI are involved. Cooperation networks between scholars are given from the figure. For instance, there is a significant collaboration between Dawn A. Sim, Ahmed Abder and Aliem Mohamed Amr (from WOS), and Zhao Xin and Yang Zhiwen (from CNKI). Fig. 3 shows that there are more collaborations between authors in CNKI than WOS.

As the Tab. 2 shown, the academic influence is measured by the amount of authors' publications. The top 2 authors are Aihong Chen (count=3) and Michael F. Chiang (count=3) from WOS, and Lin Haotian (count $=5$, centrality $=0.03$ ) and Chen Jingjing (count $=4$, centrality $=0.03$ ) from CNKI.

The Fig. 4 shows the collaboration network among institutions. 22 nodes and 20 links for WOS, and 30 nodes and 0 links for CNKI. It indicated that there are slight collaborations between institutions in WOS and no cooperation in CNKI. Collaborations between Johns Hopkins University and Kin Khalid Eye Specialist Hospital are relatively significant.

The academic influence of institutions is ranked by their publication numbers. As the tab. 4 shown, top 5 institutions in WOS are Ain Shams University (count=6, centrality=0), Sun Yat-sen University (count=6, centrality $=0$ ), Johns Hopkins University (count $=6$, centrality $=0.22$ ), Oregon Health \& Science University (count=6, centrality $=0$ ) and King Khaled Eye Specialist Hospital (count=6, centrality=0.13), two of which are in the U.S. Top 5 institutions in CNKI are Zhongshan Ophthalmology Center of Sun Yat-sen University (count $=6$, centrality $=0$ ), Beijing Tongren Hospital (count $=6$, centrality $=0$ ), the Afflated Eye Hospital of ... Nanjing Medical University (count $=6$, centrality $=0$ ), Beihang University (count $=6$, centrality $=0$ ), the company of Airdoc (count $=6$, centrality $=0$ ), three of which are in Beijing city of China.

The core journal is identified by the amount of publications. 129 nodes and 731 links are displayed in Fig. 5 According to the value of centrality, top 10 co-cited journals of WOS are listed in tab. 4 . The real-time impact factor is searched from website of Academic Accelerator, in 24th October, 2021. It indicates that publications in the top 10 journals are in a high quality. Journal of Ophthalmology is the core journal with the highest number of publications (108) and centrality (0.16).

Fig. 6 displays the distribution of cited references in WOS, 116 nodes and 380 links are given from the map. Top 5 references are recognized in Tab. 5 according to the amount of being cited. With a record of 20 of being cited, a study from Gulshan et al. (Gulshan et al. (2016)) is defined as the most academic influential article.

\section{Analysis of the hot-spots}

The hot-spot map is illustrated in Fig. 7, 64 nodes and 198 links for WOS, and 181 nodes and 562 links for CNKI are displayed. According to the Fig. 7, keywords of Artificial Intelligence, diabetic 


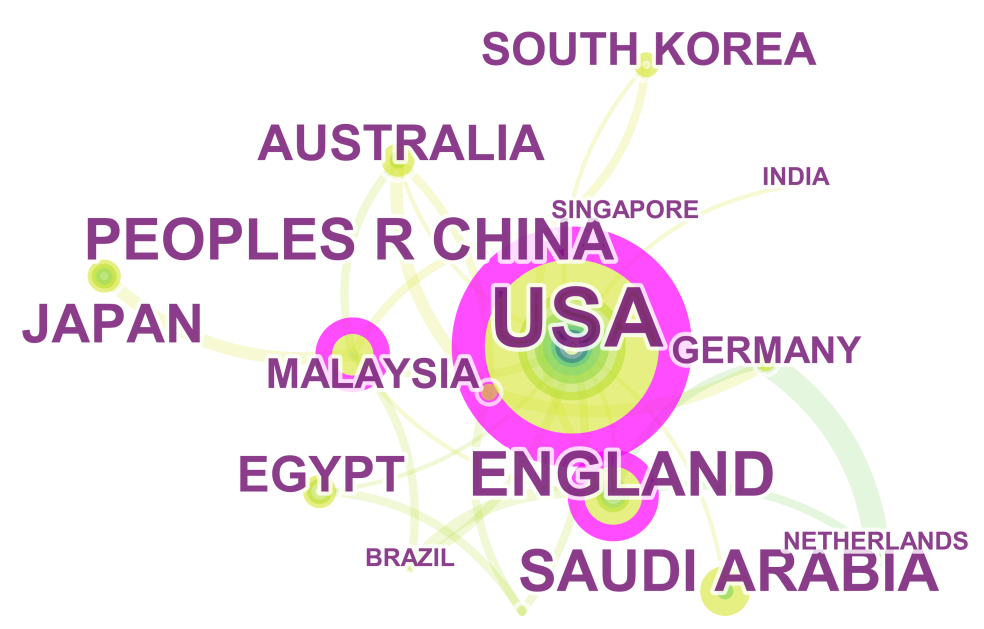

CANADA

Figure 2. The distribution of countries.

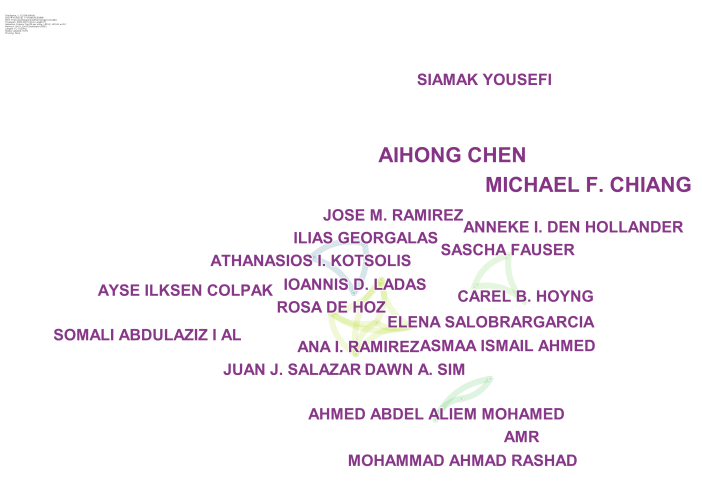

a
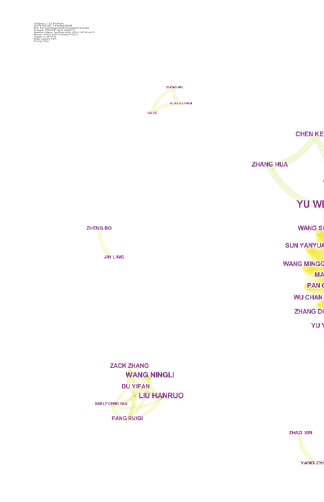

$\mathrm{b}$

Figure 3. The distribution of authors of WOS (a) and CNKI (b).

retinopathy, optical coherence tomography, deep learning, machine learning, ophthalmology, glaucoma, aged macular degeneration (AMD) and diagnosis are the most significant topics. The time zone view and the history trend of "AI in Ophthalmology" are revealed in Fig. 8 (a and c) and Fig. 9. As far as WOS database is concerned, topics of DR and AMD emerged in 2009, the keyword of OCT appeared in 2016, hot-spots of glaucoma, ML, DL and fundus image turns up in 2017 and 2018, the topic of AI burst in 2019. Regarding to the database of CNKI, the keyword of AI appeared in 2017, topics of DL, glaucoma, DR and ophthalmology emerged in 2018, AMD, ML, fundus screening and OCT-related keywords burst after 2019. Fig. 8 (b and d) presents top 12 hot-spots with strongest citation bursts of WOS and CNKI in the developing history. As for WOS database, the keyword of glaucoma draws a high attention during 2009 to 2013, the topic of OCT gains much focus during the period of 2016 to 2017, the hot-spot of AI holds the interests from 2019 to 2021 . When it comes to CNKI database, keywords of fundus screening and convolution neural network burst after 2020 .

According to the Fig. 9, topics from WOS are classified into 6 clusters, which are partial deepithelization, retinal photography, transfer learning, neuro-ophthalmology outpatient clinic, AI and age factor. It is implemented as 12 clusters for keywords of CNKI, which are AI, Digital medical technology, medical reform, hospital information security, information platform, ophthalmology, fundus image, consistency 
Table 1. Top 14 countries according to the amount of publications in WOS.

\begin{tabular}{c|c|c|c}
\hline Ranking & Country & Count & Centrality \\
\hline 1 & USA & 38 & 0.63 \\
2 & England & 14 & 0.28 \\
3 & China & 11 & 0.24 \\
4 & Saudi Arabia & 11 & 0 \\
5 & Japan & 9 & 0 \\
6 & Australia & 8 & 0.07 \\
7 & Egypt & 8 & 0 \\
8 & South Korea & 6 & 0.02 \\
9 & Malaysia & 5 & 0.05 \\
10 & Turkey & 5 & 0 \\
11 & Greece & 5 & 0 \\
12 & Germany & 4 & 0.05 \\
13 & Canada & 3 & 0.05 \\
14 & Singapore & 2 & 0.03 \\
\hline
\end{tabular}

analysis, classical hierarchical model and internet hospital.

\section{Analysis of the categories}

Based on the number of publications, the top 15 categories are listed in Fig. 10. The core discipline is defined as Ophthalmology, which is with 119 publications in WOS and 67 publications in CNKI. AI-related category emerges in the fourth place of WOS and second place in CNKI. Comparing the two databases, interdisciplinary category is creatively listed as a cluster for WOS. Some of the papers in CNKI involve the interdisciplinary subjects of AI, Ophthalmology, education and traditional Chinese medicine (TCM). The distribution of categories of WOS database is visualized in Fig. 11. 15 nodes and 9 links are involved in this figure. It reveals that corporations between computer-science-based, ophthalmology-based and engineering-based disciplines are relatively week, which should be paid more attention in the future research.

\section{DISCUSSION}

AL technology increasingly becomes a popular focus in the ophthalmology research, especially in the latest 5 years. The trend is predicted to be continually increasing in the next few years. This study utilizes the tool of CiteSpace to scientifically explore and exhibit the knowledge structure and developing trend of the specific research field of "AI in Ophthalmology".

Table 2. Top 10 authors according to the amount of publications.

\begin{tabular}{c|c|c|l|c|c|l}
\hline \multirow{2}{*}{ Ranking } & \multicolumn{3}{|c|}{ WOS } & \multicolumn{3}{c}{ CNKI } \\
\cline { 2 - 6 } & Count & Centrality & Author & Count & Centrality & Author \\
\hline 1 & 3 & 0 & Aihong Chen & 5 & 0.03 & Lin Haotian \\
2 & 3 & 0 & Michael F. Chiang & 4 & 0.03 & Chen Jingjing \\
3 & 2 & 0 & Athanasios I. Kotsolis & 3 & 0 & Chen Nan \\
4 & 2 & 0 & Ilias Georgalas & 3 & 0 & Liu Hanruo \\
5 & 2 & 0 & Ioannis D. Ladas & 3 & 0 & Du Yifan \\
6 & 2 & 0 & Ahmed Abdel Aliem Mohamed & 3 & 0 & Wang Ningli \\
7 & 2 & 0 & Asmaa Ismail Ahmed & 3 & 0 & Yang Weihua \\
8 & 2 & 0 & Mohammad Ahmad Rashad & 3 & 0 & Zack Zhang \\
9 & 2 & 0 & Anneke I. Den hollander & 2 & 0 & Zhang Xiao \\
10 & 2 & 0 & Carel B. Hoyng & 2 & 0 & Pang Ruiqi \\
\hline
\end{tabular}




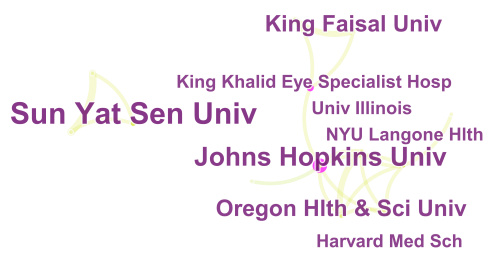

Ain Shams Univ

a

Figure 4. The distribution of institutions of WOS (a) and CNKI (b).

Table 3. Top 10 authors according to the amount of publications.

\begin{tabular}{|c|c|c|c|c|c|c|c|c|}
\hline \multirow{2}{*}{ Ranking } & \multicolumn{4}{|c|}{ WOS } & \multicolumn{4}{|c|}{ CNKI } \\
\hline & Institution & Country & Count & Centrality & Institution & City of China & Count & Central \\
\hline 1 & $\begin{array}{l}\text { Ain Shams } \\
\text { University }\end{array}$ & Egyptian & 6 & 0 & $\begin{array}{l}\text { Zhongshan } \\
\text { Ophthalmology } \\
\text { Center } \\
\text { of Sun Yat-sen } \\
\text { University }\end{array}$ & Guangzhou & 7 & 0 \\
\hline 2 & $\begin{array}{l}\text { Sun Yat-sen } \\
\text { University }\end{array}$ & China & 5 & 0 & $\begin{array}{l}\text { Beijing Tongren } \\
\text { Hospital }\end{array}$ & Beijing & 7 & 0 \\
\hline 3 & $\begin{array}{l}\text { Johns Hopkins } \\
\text { University }\end{array}$ & The U.S. & 4 & 0.22 & $\begin{array}{l}\text { Eye Hospital } \\
\text { of Nanjing } \\
\text { Medical } \\
\text { University }\end{array}$ & Nanjing & 5 & 0 \\
\hline 4 & $\begin{array}{l}\text { Oregon Health } \\
\& \text { Science } \\
\text { University }\end{array}$ & The U.S. & 3 & 0 & $\begin{array}{l}\text { Beihang } \\
\text { University }\end{array}$ & Beijing & 3 & 0 \\
\hline 5 & $\begin{array}{l}\text { King Khaled } \\
\text { Eye Specialist } \\
\text { Hospital }\end{array}$ & $\begin{array}{l}\text { Saudi } \\
\text { Arabia }\end{array}$ & 3 & 0.13 & $\begin{array}{l}\text { The company } \\
\text { of Airdoc }\end{array}$ & Beijing & 2 & 0 \\
\hline
\end{tabular}

\section{Data analysis}

Topic mapping and its time distribution are displayed in this article. As the fig. 7 shown, DR, AMD, glaucoma, AI, OCT, fundus image, DL, ML, diagnosis are the major topics in both databases of the WOS and CNKI. Thus, this study indicates that the application of AI in Ophthalmic diagnosis with OCT fundus images plays a significant role in the "AI in Ophthalmology" studies, especially for the detection of DR, AMD and glaucoma. It is also a vital research direction in the following years.

Furthermore, scientific publications are identified as one indicator of core competitiveness of a country (Wu et al. (2020); Liang et al. (2018); Sun et al. (2021)). As the Tab. 1. and Fig. 2. shown, according to the country distribution result of WOS, the USA shows an competitive advantage in this research field, followed by England and China. According to the Tab. 3 and Fig. 4, with the most number of publications, institutions of Ain Shams University (Egyptian), Sun Yat-sen University (in China) and Johns Hopkins University (in the U.S.) are regarded as the top-three institutions in this research area. However, measured by the centrality result ( $>0.1$ ), Johns Hopkins University (the U.S.) and King Khalid Eye Specialist Hospital (Saudi Arabia) are the most essential institutions. When it comes to CHKI, most of institutions involved are from China, where Zhongshan Ophthalmology center of Sun Yat-sen University and Beijing Tongren Hospital presents a significant contribution academically.

Besides, the academic influence of articles, authors and journals can be determined by the cited 


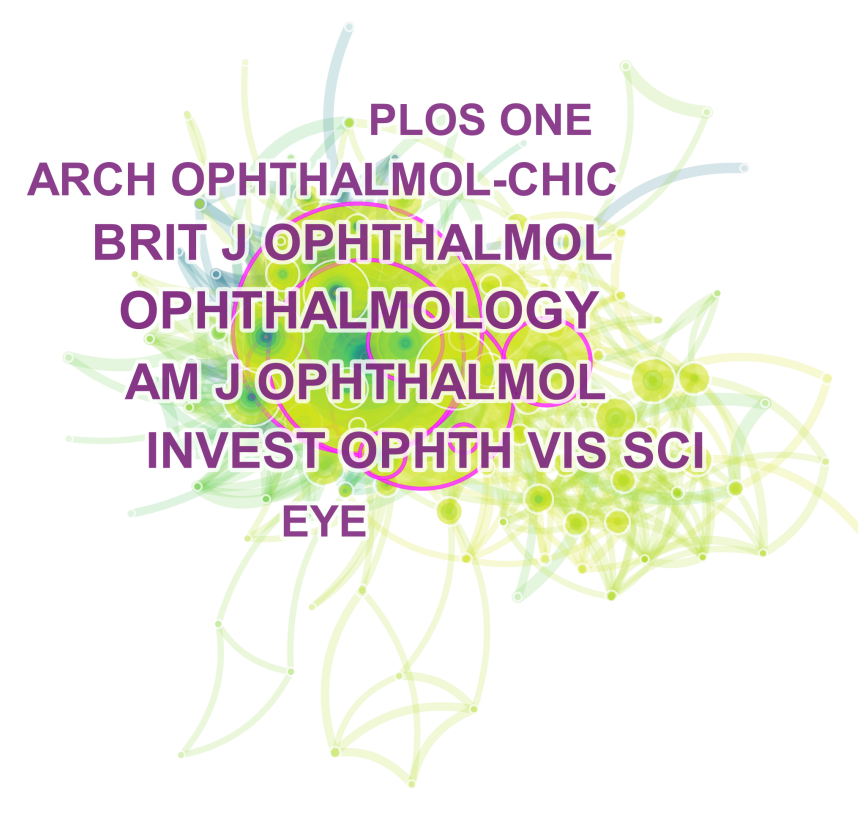

Figure 5. The distribution of cited journal of WOS.

frequency and the centrality result ( $>0.1)$ (Sun et al. (2021)). As the Tab. 5 and Fig. 6 shown, regarding to WOS database, the article of "Development and validation of a deep learning algorithm for detection of diabetic retinopathy in retinal fundus photographs" (Gulshan et al. (2016)) exhibits the greatest advantage in WOS database, it possesses the highest cited amount (20) and a relatively high centrality value $(0.12)$. As the Fig. 3, Fig. 5, Tab. 2 and Tab. 4 shown, Aihong Chen is the most influential author, who is involved to the research of comparing the vision screening programs within 18 countries (Chen et al. (2019)). Journal of Ophthalmology is the core journal with the highest cited record (108) and a centrality value (0.16). As far as CNKI is concerned, Haotian Lin presents the most advantage in publication frequency and centrality value, who is involved in the paper named as "Establishment of ophthalmic data center and intelligent service cloud platform", which is a research related to the applications of AI to intelligent ophthalmic center constructions (pisong et al. (2021)).

The number of nodes and links in cooperative network indicates the cooperative relationships, where the higher the number of links, the stronger the relation is (Liang et al. (2018); Sun et al. (2021); Wang et al. (2018)). According to the social network of WOS exhibited in Fig. 3. (note $=19$, link=25 for WOS; note $=239$, link $=619$ for CNKI), Fig. 4. (note $=22$, link=20 for WOS; note $=30$, link=0 for CNKI) and fig. 2. (note $=19$, line $=25$ for WOS), the cooperation between authors, institutions and countries are insufficient. Moreover, as the Fig. 11 (node=15, link=9) shown, the research of Interdisciplinary theories and applications should be paid more attention.

The strength of bursting citation of keywords represents the degree of retrieving focuses in the specific period (Li et al. (2018)). By analyzing the Fig. 8 and Fig. 9, it is indicated that AI-diagnose-related keywords are not significant enough in the developing history line before 2019, which presents a huge burst during 2019 to 2021. The topic of OCT fundus image exhibits a great burst in 2016 (from WOS) and 2020 (from CNKI).

Comparing the database of WOS and CNKI, there is a significant difference in the aspects of developing histories and discipline categories. As Fig. 1, Fig. 8 and Fig. 9 shown, this study concludes that the development of the AI in Ophthalmology research of the WOS database is slightly advanced 
Table 4. The top 10 co-cited journals of WOS according to centrality.

\begin{tabular}{c|c|c|c|c}
\hline Ranking & Count & Centrality & Journal & Real-time Impact factor \\
\hline 1 & 108 & 0.16 & Ophthalmology & 14.8 \\
2 & 80 & 0.16 & Investigative Ophthalmology and Visual Science & 6.6 \\
3 & 46 & 0.15 & Eye & 5 \\
4 & 20 & 0.15 & Acta Ophthalmologica & 5.2 \\
5 & 14 & 0.15 & Scientific Reports & 7.1 \\
6 & 29 & 0.13 & Survey of Ophthalmology & 8.5 \\
7 & 88 & 0.12 & American Journal of Ophthalmology & 7.1 \\
8 & 33 & 0.12 & Acta Ophthalmologica & 5.2 \\
9 & 37 & 0.1 & Journal of the American Medical Association & 56.272 \\
10 & 18 & 0.1 & New England Journal of Medicine & 74.699 \\
\hline
\end{tabular}
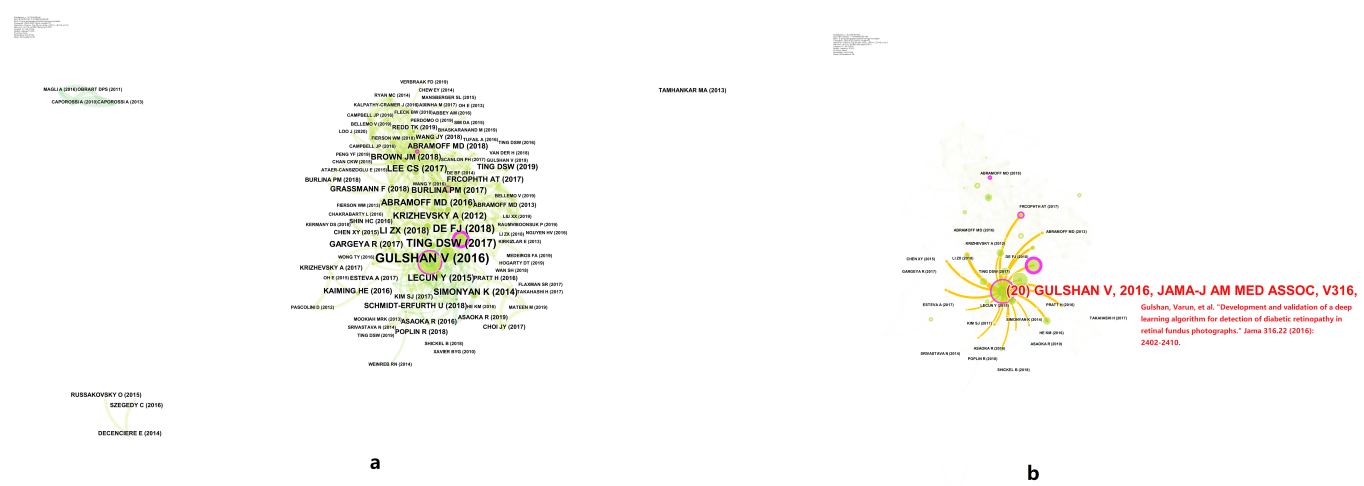

Figure 6. The distribution of cited reference of WOS.

than CNKI. CNKI's developing history starts with the bursting topic of "robot" (2014), which brings "AI" (2017) two years early than WOS (2019). Hot-topic of "diabetic retinopathy" (2009) is the beginning in WOS's developing history, which emerges in 2018 for CNKI. The keyword of "optical coherence tomography "bursts in 2016 for WOS, which is three years early than CNKI (2019). Thus, considering bursting topics and time series, this study divides the developing history of the two databases into three phases. As far as WOS studies are concerned, the first, second and the last phase is defined as "fundus disease" (2009)," OCT" (2016) and "AI" (2019) respectively. As for the CNKI database, three phases are recognized as "AI" (2014), "fundus disease" (2018) and "OCT" (2019). Moreover, the Fig. 7indicates that the cross degree of WOS's interdisciplinary research is more significant than CNKI, especially for AI and OCT studies. As the Fig. 10 shown, ophthalmology-based, engineering-based and computer-based disciplines are involved in both of the databases. Category of traditional Chinese medicine (TCM) is a novel discipline of CNKI for "AI in ophthalmology" (Hang et al. (2021)). In their studies, they classified the AI-based TCM studies into four categories, which are TCM diagnosis, TCM syndrome differentiation, health management and ophthalmology. When it comes to the aspects of ophthalmology, the paper highlights two studies from Wen (Yi (2016)) and Li (Ruiling (2012)) for their contributions of TCM eye observation. (Yi (2016)) performs a research on iris diagnosis with computer vision algorithms (Hang et al. (2021)). Li proposes DL algorithms of eye edge extraction and pupil center location (Hang et al. (2021); Ruiling (2012)).

\section{Strengths and limitations}

This research is regarded as the first study to systematically explore the literature from 2000 to 2021 on applications of AI (ML and DL) to ophthalmology. Papers of WOS and CNKI in Chinese and English languages are analyzed in this paper. The information of leading authors, journals, institutions, nations and references is identified. Insights of potential cooperative relationship among authors, institutions and countries are provided. Findings of the hot-spot mapping, developing history and future research direction 
Table 5. The top 10 co-cited journals of WOS according to centrality.

\begin{tabular}{|c|c|c|c|c|c|}
\hline Ranking & Count & Centrality & Year & Cited Reference & Author \\
\hline 1 & 20 & 0.12 & 2016 & $\begin{array}{l}\text { "Development and Validation of } \\
\text { a Deep Learning Algorithm } \\
\text { for Detection of Diabetic } \\
\text { Retinopathy in Retinal Fundus } \\
\text { Photographs" }\end{array}$ & $\begin{array}{c}\text { Gulshan et al. } \\
\text { (Gulshan et al. (2016)) }\end{array}$ \\
\hline 2 & 13 & 0.04 & 2017 & $\begin{array}{l}\text { "Development and Validation } \\
\text { of a Deep Learning System } \\
\text { for Diabetic Retinopathy } \\
\text { and Related Eye Diseases } \\
\text { Using Retinal Images from } \\
\text { Multiethnic Populations } \\
\text { with Diabetes" }\end{array}$ & $\begin{array}{c}\text { Ting et al. } \\
\text { (Ting et al. (2017)) }\end{array}$ \\
\hline 3 & 11 & 0.27 & 2018 & $\begin{array}{l}\text { "Clinically Applicable Deep } \\
\text { Learning for Diagnosis and } \\
\text { Referral in Retinal Disease" }\end{array}$ & $\begin{array}{c}\text { De Fauw et al. } \\
\text { (De Fauw et al. (2018)) }\end{array}$ \\
\hline 4 & 8 & 0.05 & 2012 & $\begin{array}{l}\text { "ImageNet Classification with } \\
\text { Deep ConvolutionalNeural } \\
\text { Networks" }\end{array}$ & $\begin{array}{l}\text { Krizhevsky, Sutskever, } \\
\text { \& Hinton } \\
\text { (Krizhevsky et al. (2012)) }\end{array}$ \\
\hline 5 & 8 & 0.04 & 2018 & $\begin{array}{l}\text { Learning System for Detecting } \\
\text { Glaucomatous Optic Neuropathy } \\
\text { Based on Color Fundus } \\
\text { Photographs" }\end{array}$ & $\begin{array}{c}\text { Li et al. } \\
\text { (Li et al. (2018)) }\end{array}$ \\
\hline
\end{tabular}

are contributed. Finally, a comparison of "AI in Othalmology" studies between WOS and CNKI datasets is discussed. The potential academic value of interdisciplinary subject of "AI in Ophthalmology" and TRM is discussed. Directions and suggestions for the future research are indicated.

However, limitations are inevitable. This study is performed based on papers retrieved from WOS and CNKI on the specific keyword searching. It should be considered to applying more related searching strings in multiple databases for more literature. Besides, this study only considered Chinese and English articles, it is suggested to perform bibliographic research on papers in more than two languages.

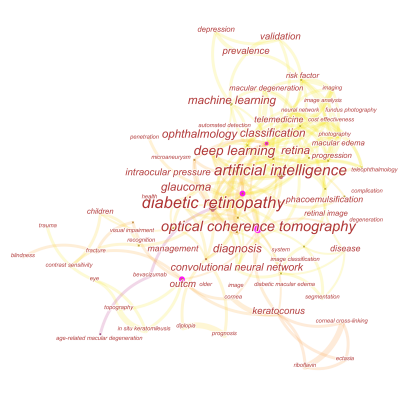

a

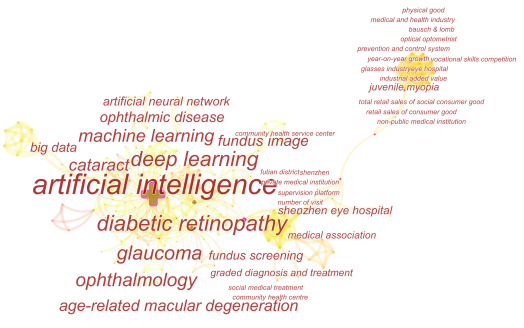

b

Figure 7. The topic mapping visualization of WOS (a) and CNKI (b). 


\section{CONCLUSION}

AI-based technologies exhibit a great potential value for Ophthalmology. Although there is a difference in developing history between WOS and CNKI, the future direction extracted from the two databases is as the same as the application of AI in Ophthalmic diagnose with OCT fundus images. Furthermore, results indicates that the traditional Chinese medicine applied to this area could be another future direction. Moreover, deeper cooperation between authors, institutions and countries of interdisciplinary studies should be enhanced to promote the research of "AI in Ophthalmology".

\section{ACKNOWLEDGMENTS}

This research was funded by the 2020 Key Technology R\&D Program of GuangDong Province, grant number ZH01110405180056PWC, Zhuhai Technology and Research Foundation, grant number ZH01110405180056PWC, Zhuhai Basic and Application Research Project, grant number ZH22017003200011PWC and Zhuhai Basic and Application Research Project, grant number ZH22036201210034PWC.

\section{REFERENCES}

Cen, L.-P., Ji, J., Lin, J.-W., Ju, S.-T., Lin, H.-J., Li, T.-P., Wang, Y., Yang, J.-F., Liu, Y.-F., and Tan, S. (2021). Automatic detection of 39 fundus diseases and conditions in retinal photographs using deep neural networks. Nature communications, 12(1):1-13.

Chen, A.-H., Bakar, N. F. A., and Arthur, P. (2019). Comparison of the pediatric vision screening program in 18 countries across five continents. Journal of current ophthalmology, 31(4):357-365.

Dai, L., Wu, L., Li, H., Cai, C., Wu, Q., Kong, H., Liu, R., Wang, X., Hou, X., and Liu, Y. (2021). A deep learning system for detecting diabetic retinopathy across the disease spectrum. Nature communications, 12(1):1-11.

De Fauw, J., Ledsam, J. R., Romera-Paredes, B., Nikolov, S., Tomasev, N., Blackwell, S., Askham, H., Glorot, X., O’Donoghue, B., Visentin, D., van den Driessche, G., Lakshminarayanan, B., Meyer, C., Mackinder, F., Bouton, S., Ayoub, K., Chopra, R., King, D., Karthikesalingam, A., Hughes, C. O., Raine, R., Hughes, J., Sim, D. A., Egan, C., Tufail, A., Montgomery, H., Hassabis, D., Rees, G., Back, T., Khaw, P. T., Suleyman, M., Cornebise, J., Keane, P. A., and Ronneberger, O. (2018). Clinically applicable deep learning for diagnosis and referral in retinal disease. Nature Medicine, 24(9):1342-1350.

Gulshan, V., Peng, L., Coram, M., Stumpe, M. C., Wu, D., Narayanaswamy, A., Venugopalan, S., Widner, K., Madams, T., and Cuadros, J. (2016). Development and validation of a deep learning algorithm for detection of diabetic retinopathy in retinal fundus photographs. Jama, 316(22):2402-2410.

Hang, W., Li, H., Jiang, L., and Minhong, X. (2021). Research progress of artificial intelligence technology in clinical diagnosis and treatment of traditional chinese medicine. China Medical Herald, 18:42-45.

Krizhevsky, A., Sutskever, I., and Hinton, G. E. (2012). ImageNet Classification with Deep Convolutional Neural Networks, volume 25. Curran Associates, Inc.

Li, Z., He, Y., Keel, S., Meng, W., Chang, R. T., and He, M. (2018). Efficacy of a deep learning system for detecting glaucomatous optic neuropathy based on color fundus photographs. Ophthalmology, 125(8):1199-1206.

Liang, C., Luo, A., and Zhong, Z. (2018). Knowledge mapping of medication literacy study: a visualized analysis using citespace. SAGE open medicine, 6:2050312118800199.

Marta, W.-M., Andrzej, O., Marta, B.-W., Aleksandra, B., and Gołebiewska, J. (2021). Choroidal thickness in children with type 1 diabetes depending on the pubertal status and metabolic parameters analyzed by optical coherence tomography.

pisong, Y., Yifan, X., Qiang, L., Jingjing, C., and Haotian, L. (2021). Establishment of ophthalmic data center and intelligent service cloud platform. Eye Science, 36(1000-4432):1-3.

Ran, A. R., Tham, C. C., Chan, P. P., Cheng, C.-Y., Tham, Y.-C., Rim, T. H., and Cheung, C. Y. (2021). Deep learning in glaucoma with optical coherence tomography: A review. Eye, 35(1):188-201.

Ruiling, L. (2012). Research on objectification of facial appearance in traditional Chinese medicine based on computer vision. Thesis.

Scheetz, J., Rothschild, P., McGuinness, M., Hadoux, X., Soyer, H. P., Janda, M., Condon, J. J., OakdenRayner, L., Palmer, L. J., and Keel, S. (2021). A survey of clinicians on the use of artificial intelligence in ophthalmology, dermatology, radiology and radiation oncology. Scientific reports, 11(1):1-10. 
Shao, E., Liu, C., Wang, L., Song, D., Guo, L., Yao, X., Xiong, J., Wang, B., and Hu, Y. (2021). Artificial intelligence-based detection of epimacular membrane from color fundus photographs. Scientific reports, 11(1):1-10.

Sooryamoorthy, R. (2020). Scientometrics for the study of sociology. International Sociology, 35(5):461479.

Sun, Y., Li, C., Zhao, Y., and Sun, J. (2021). Trends and developments in oral health literacy: a scientometric research study (1991-2020). BDJ open, 7(1):1-10.

Ting, D. S. W., Cheung, C. Y.-L., Lim, G., Tan, G. S. W., Quang, N. D., Gan, A., Hamzah, H., GarciaFranco, R., San Yeo, I. Y., Lee, S. Y., Wong, E. Y. M., Sabanayagam, C., Baskaran, M., Ibrahim, F., Tan, N. C., Finkelstein, E. A., Lamoureux, E. L., Wong, I. Y., Bressler, N. M., Sivaprasad, S., Varma, R., Jonas, J. B., He, M. G., Cheng, C.-Y., Cheung, G. C. M., Aung, T., Hsu, W., Lee, M. L., and Wong, T. Y. (2017). Development and validation of a deep learning system for diabetic retinopathy and related eye diseases using retinal images from multiethnic populations with diabetes. JAMA, 318(22):2211-2223.

Wang, M., Xiao, C., Ni, P., Yu, J.-J., Wang, X.-W., and Sun, H. (2018). Correlation of betel quid with oral cancer from 1998 to 2017: a study based on bibliometric analysis. Chinese medical journal, 131(16): 1975.

Wu, M., Wang, Y., Yan, C., and Zhao, Y. (2020). Study on subclinical hypothyroidism in pregnancy: a bibliometric analysis via citespace. The Journal of Maternal-Fetal Neonatal Medicine, pages 1-12.

Yang, J., Fong, S., Wang, H., Hu, Q., Lin, C., Huang, S., Shi, J., Lan, K., Tang, R., and Wu, Y. (2021). Artificial intelligence in ophthalmopathy and ultra-wide field image: a survey. Expert Systems with Applications, page 115068.

Yi, W. (2016). Research on digital method of TCM visual diagnosis based on image analysis. Thesis. 

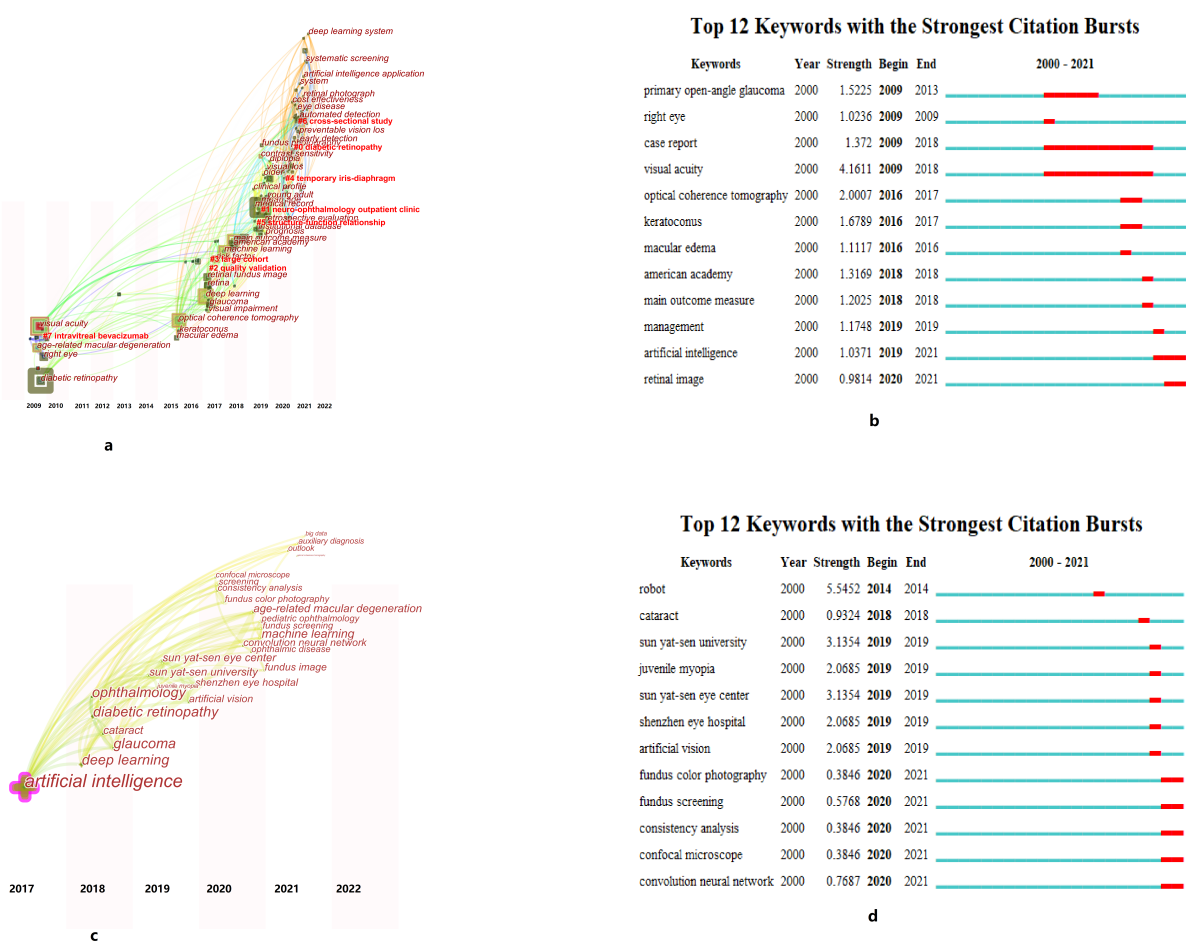

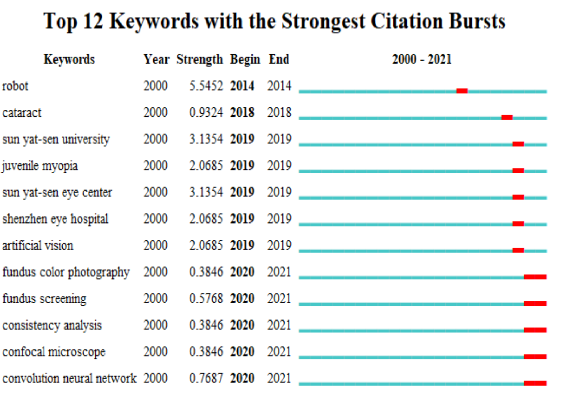

Figure 8. The time zone and the citation bursts of keywords of WOS (a and b) and CNKI (c and d).

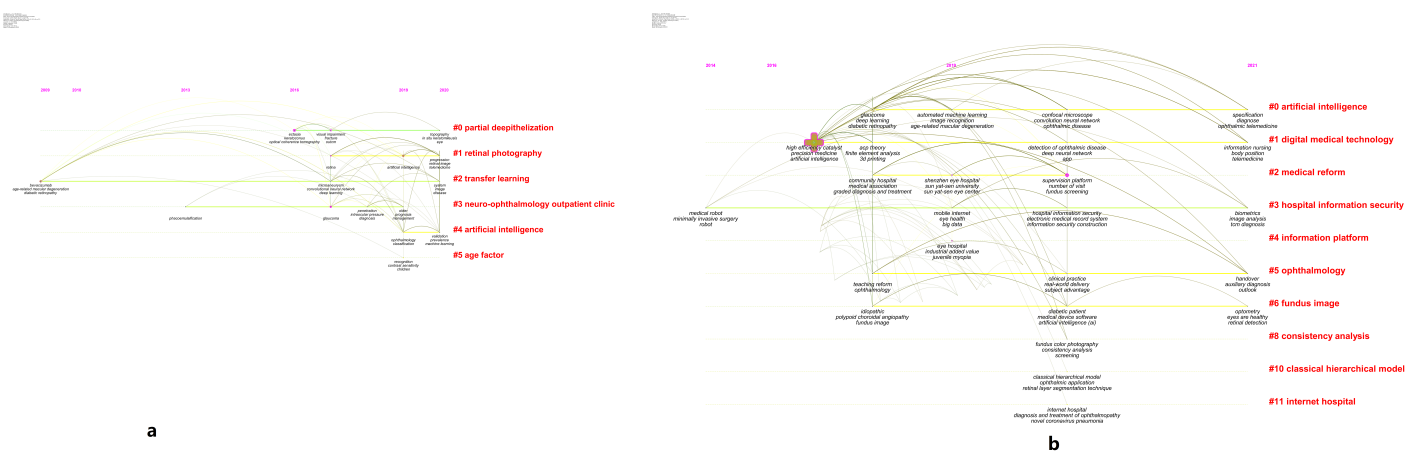

Figure 9. Topic developing history and cluster visualization of WOS (a) and CNKI (b).

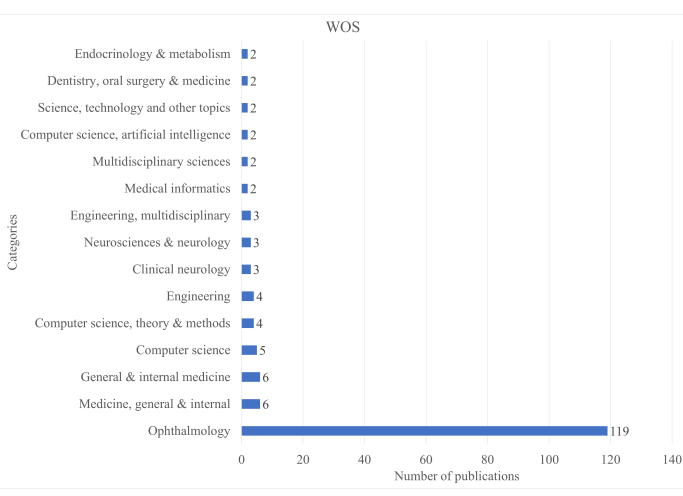

a

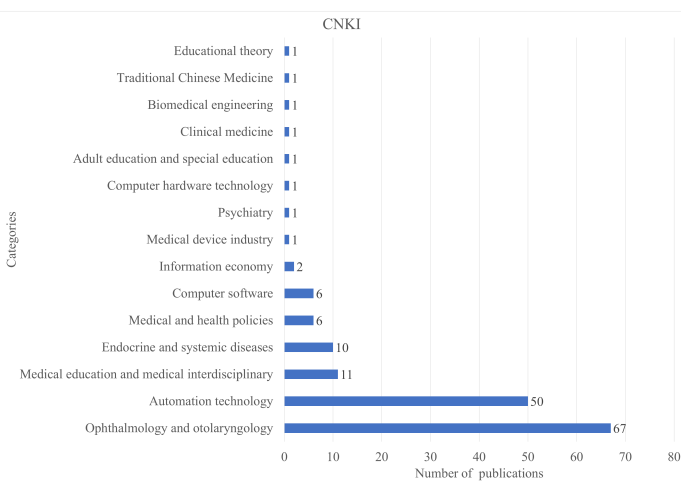

b

Figure 10. Top 15 categories according to the amount of publications of WOS (a) and CNKI (b). 


\section{DENTISTRY, ORAL SURGERY \& MEDICINE \\ MEDICINE, GENERAL \& INTERNAL

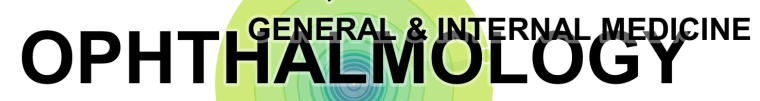 \\ CLINICAL NEUROLOGYENGINEERINGMEDICAL INFORMATICS ENGINEERING, MULTIDISCIPLINARY NEUROSCIENCES \& NEUROLOGY \\ MULTIDISCIPLINARY SCIENCES \\ COMPUTER SCIENCE, THEORY \& METHODS \\ SCIENCE \& TECHNOLOGY - OTHER TOPICS \\ COMPUTER SCIENCE, ARTIFICIAL INTELLIGENCE \\ COMPUTER SCIENCE}

Figure 11. The distribution of categories of WOS database. 